\title{
Research and Realization of Operation Monitoring System for Electric Vehicle Fast Charging Station
}

\author{
Chang Liu ${ }^{1, a}$, Bin $\mathrm{Li}^{1, \mathrm{~b}}$, Jing Zhang ${ }^{1, \mathrm{c}}$, Lu Yu ${ }^{2, \mathrm{~d}}$ and Danping Zou ${ }^{3, \mathrm{e}}$ \\ ${ }^{1}$ China Electric Power Research Institute, Beijing Electric Vehicle Charging/Battery Swap \\ Engineering and Technology Research Center, Beijing 100085, China \\ ${ }^{2}$ School of Control and Computer Engineering, North China Electric Power University, Beijing \\ 102206, China \\ ${ }^{3}$ Beijing Puri High-voltage Transmission Technology Co., Ltd of State Grid, Beijing 102208, China \\ aliuchang3@epri.sgcc.com.cn, blibin5@epri.sgcc.com.cn, copkl_5606@163.com, \\ dyulufly2009@163.com, èzoudanping@sgepri.sgcc.com.cn
}

Keywords: Operation monitoring system for electric vehicle fast charging station, design of system architecture, business functional design, communication design

\begin{abstract}
Building a fast charging network puts forward new demands of operation monitoring system for electric vehicle fast charging station, through investigation of different situations for fast charging station, study business needs of fast charge stations under different situations, and do system architecture, business functional design and communication design. Fast charging station operation monitoring system's business modules mainly include vehicle management, payment, metering and billing, smart card management and end-user services. Research and development of fast charging station operation monitoring system can provide technical support for the operation of fast charging stations.
\end{abstract}

\section{Introduction}

According to development strategy and electricity supplementation demand of electric vehicle in China, fast charging mode will become an important mode of energy supply of electric vehicle[1]. Construction of electric vehicle fast charging network and promotion of scaled development of electric vehicles are important approaches to implement energy saving and emission reduction policy, reduce air pollution and control haze and are important measures to implement electric energy substitution and promote the fuel substitution by electricity ${ }^{[2]}$.

Construction of urban public fast charging network and intercity interconnection fast charging network puts forward new demands of operation monitoring system for electric vehicle fast charging station which require overall and full process guidance of electric vehicle fast charging, improvement of user experience in fast charging mode, promotion of innovation of operation mode of electric vehicle charging facilities in fast charging mode and promotion and application of electric vehicle fast charging mode ${ }^{[3]}$.

\section{Structure Design of Operation Monitoring System for Electric Vehicle Fast Charging Station}

The electric vehicle fast charging stations mainly include urban public fast charging stations (including three dimensional charging tower and three dimensional parking charging lot) and intercity interconnection fast charging stations. Basic business covers parking, guiding, charging and fee collecting. The fast charging station, based on business demand, provides parking service, charging service, metering and billing, automatic guidance, etc.

Operation monitoring system for fast charging station has equipment layer, spacing layer and station control layer. The system hierarchical structure diagram is figure 1. Station monitoring system 
interface is supported. It can be connected with operation management system and obtain metering and billing information and provide spot price ${ }^{[5]}$.

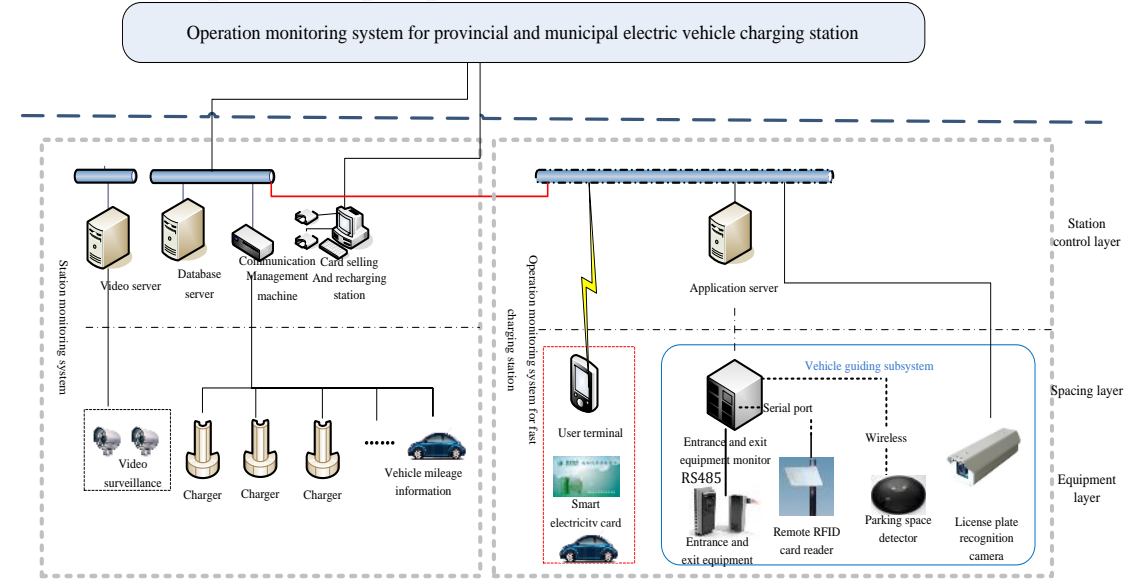

Fig. 1 System hierarchical structure diagram

1) Equipment layer: be mainly composed of bottom hardware devices, including user smart terminal (such as mobile phone), smart electricity card, vehicle smart terminal, entrance and exit equipment controller, remote RFID card reader, parking space detector, LED guiding panel, license plate recognition camera and other entrance and exit equipment ${ }^{[6]}$.

2) Spacing layer: be composed of control modules of sub-systems, including identification module, metering and billing module, vehicle guiding module and smart card management module.

3) Station control layer: be established on the top layer and conduct coordination control of all sub-modules of spacing layer. Application server in station control layer is equipped with software and database of operation monitoring system. The station control layer conducts management and remote control of hardware equipment in equipment layer via spacing layer control module and carries out process analysis and storage of data generated in functional sub-modules ${ }^{[7]}$. Besides, station control layer acquires charging data of station monitoring system and upper layer provincial operation monitoring system via WebService interface and applies data in different functions.

\section{Design of Operation Monitoring System for Electric Vehicle Fast Charging Station}

Operation monitoring system for fast charging station has five business modules, including vehicle management, payment, metering and billing, smart card management and end-user services.

\subsection{Vehicle Management}

The vehicle management module mainly includes identification, dynamic parking space allocation, vehicle guidance, parking management and other functions.

1) Identification: when the vehicle enters the station, RFID card reader can read the user's identity and vehicle information via smart card and identify vehicle license plate information and compare the information by license plate recognition camera to realize vehicle identification.

2) Dynamic parking space allocation and vehicle guidance: smart parking space guidance function of fast charging station can rapidly provide parking space guiding service for entering vehicles. The vehicle guidance is mainly applied in parking lot-type fast charging stations in urban area. In general, the expressway and public transport fast charging stations are special charging stations. The charging equipment is less. Therefore, vehicle guidance isn't required.

\subsection{Payment Management}

Payment module mainly includes payment and recharge, charge transfer, integrated payment and refund.

Payment and recharge: recharge the smart electricity card by the payment and recharge module.

Charge transfer: realize reconciliation and settlement of all charging and parking fees in fast charging station. 
Integrated settlement payment: payment strategies are slightly different in different application situations. The expressway and public transport fast charging stations are special charging stations. The user expects to use the vehicle as soon as possible after charging in expressway service areas, public transport fast charging stations and taxi fast charging stations. Such users only need to pay the charging fees. As to fast charging stations, charging towers and three dimensional charging stations in parking lot in urban area, most users are private car owners and have dual demand of charging and parking. Therefore, aimed at such application situation, in order to provide better user experience and realize payment of both charging and parking, the parking fees will not be charged within the charging period (or reduced). If the vehicle fails to drive out of the parking lot within specified period after charging, the parking fees shall be charged according to regulation.

(1) Automatic integrated fee settlement of expressway and public transport fast charging station

Fees of charging are paid by card. Electric vehicle user can pay the charging fees via smart card provided by operation enterprise. The specific flow is shown in figure 2 .

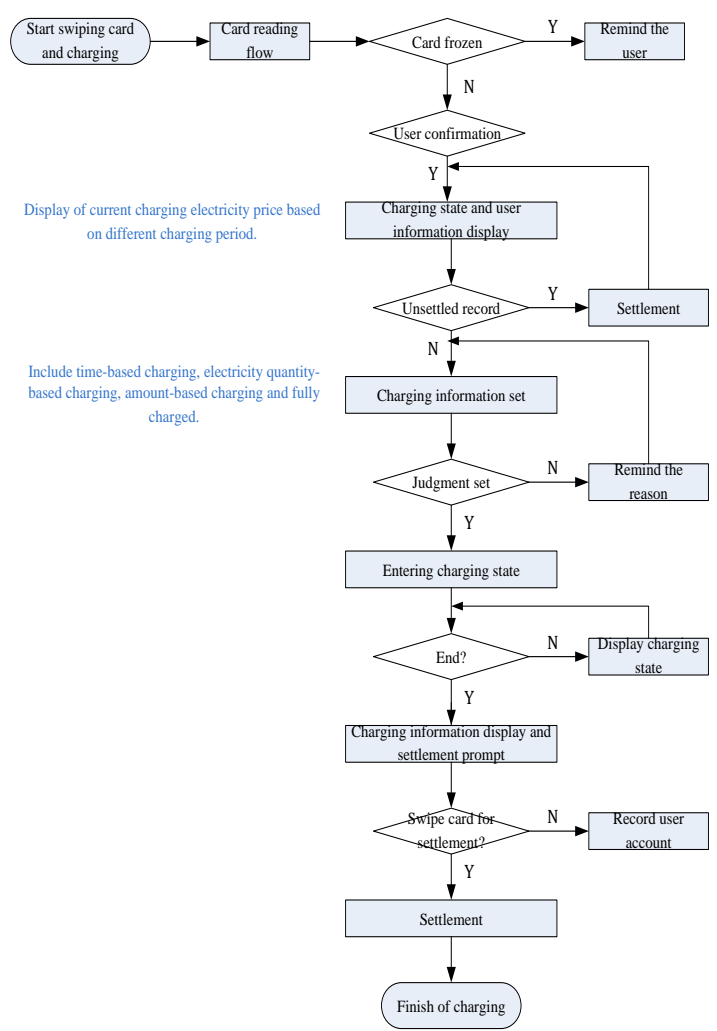

Figure 2 Billing and paying flow of expressway/public transport fast charging station

(2) Automatic integrated fee settlement of urban public fast charging station

Fees of charging are also paid by card. Electric vehicle user can pay the charging and parking fees via smart card provided by operation enterprise or temporary card obtained during entering the station. The specific flow is as follows:

a. When the vehicle enters the parking lot, RFID card reading system will read the smart charging card. The vehicle guiding system will allocate the charging space based on current charging space situation and allocation strategy of charging space;

b. When the vehicle drives to the charging space, swipe the card to set and start charging. Charging pile will display the charging state and fees;

c. After charging, user's mobile phone will receive the reminding of charging finish.

d. The vehicle leaves the parking lot. The operation monitoring system will calculate integrated charging and parking fees. The user can pay the fees by one time.

\subsection{Metering and Billing}

Metering and billing module mainly includes billing model management, billing adjustment, fee calculation, metering management and other modules. 
1) Influence factors of charging billing

Influence of charging electricity fees of electric vehicle in different development stages (early, middle and matured period), different working features (holidays and working days) and different time (day or night and peak or valley) shall be considered.

Period-based: according to different development stages of electric vehicles, including early, middle and matured period, governments may introduce relevant electricity fee policies to encourage new energy electric vehicles. Besides, operation companies can flexibly set the influence coefficients so as to attract user resources and take part in market competition well.

Time-based: articles of electricity fees at peak or valley of grid company or local government shall be considered to adjust the charging fees.

Working feature-based: set the electricity fee strategy at different time periods in holidays and working days.

Caused by uncertainty of time and space of electric vehicle as charging load, scaled electric vehicle charging certainly will generate load impact to grid and bring new challenge to operation and control. The period-based and time-based billing encourages users to orderly charge in period with low electricity fees and will relieve pressure from scaled electric vehicle on grid at certain degree[8].

2) Period-based and time-based billing model of expressway/public traffic fast charging station

Only the charging fee is available to expressway and public traffic fast charging stations. In period-based and time-based billing model of such charging stations, current charging electricity fee is product of time-based preferential billing coefficient and sum of product of charging electricity quantity and electricity price in different time.

Current charging fee $=$ time-based preferential billing coefficient $*$ (spike electricity price* spike electricity quantity + peak electricity price * peak electricity quantity+ common electricity price* common electricity quantity+ valley electricity price *valley electricity quantity+ ridge valley electricity price * ridge valley electricity quantity).

3) Period-based and time-based billing model of urban fast charging station

Fees of urban fast charging stations include charging and parking fee. In period-based and time-based billing model of such charging stations, the fee is sum of current charging fee (product of time-based preferential billing coefficient and sum of product of charging electricity quantity and electricity price in different time) and parking fee.

Current charging fee $=$ time-based preferential billing coefficient $*$ (spike electricity price* spike electricity quantity + peak electricity price $*$ peak electricity quantity+ common electricity price* common electricity quantity+ valley electricity price *valley electricity quantity+ ridge valley electricity price $*$ ridge valley electricity quantity) +parking fee.

Metering standard of parking fee is free-parking in charging period and billing in case of no charging or after charging based on collection standard.

5) Billing adjustment

The billing system can be adjusted (increase or reduce) in case of error of user file, metering equipment, electricity quantity fed back by charging station or charging equipment, received billing adjustment order and internal billing. Billing adjustment business includes application, review, approval and release of billing adjustment.

\subsection{Smart card management}

The smart card, as an effective approach of charging, paying and identification, can provide more convenient charging and paying business service for users and improve the user's experience. Smart card management mainly includes recharging, selling, changing, locking, inquiry, reissuing, loss report, unlocking, hanging solutions and refund of card, etc.

\subsection{End-user service}

Services, such as charging information query, charging state query, charging finish reminding, information announcement, service guide and personal information can be provided to users. In order to meet user experience well, information announcement function is provided. When the charging is finished, the background will send the reminding message to user's phone to inform the finish of charging. 


\section{Conclusion}

Operation monitoring system for electric vehicle fast charging station which supports fast charging mode can monitor state and information of electric vehicle and charging facilities and provide charging state query, charging finish reminding, dynamic charging space allocation, card management, identification and automatic guidance for users. When the vehicle passes the exit, the fees are charged at one time. Overall and full process guidance of fast charging of electric vehicle is realized. Therefore, operation monitoring system for electric vehicle fast charging station can greatly improve user's experience and service capacity of electric vehicle fast charging station.

With planning and building electric vehicle fast charging network, the operation monitoring system for electric vehicle fast charging station provides optimal scheme to optimize operation mode and economic benefit of electric vehicle charging facilities and has wide development space and application prospect.

\section{Acknowledgements}

This work was supported by a grant from the state grid technology program about the key techniques of electric vehicle charging infrastructure operation under the fast charging mode (No. YD71-15-032).

\section{References}

[1]Wenliang Zhang, Bin Wu, Wufeng Li, Xiaokang Lai, et al. Discussion on Development Trend of Battery Electric Vehicles in China and its Energy Supply Mode [J]. Power System Technology. 2009(04)

[2]Liangliang Chen, Hao Zhang, Feng Ni, Jinda Zhu, et al. Discussion on Current Construction Situation and Development of Electric Vehicle Energy Supply Infrastructure [J]. Automation of Electric Power Systems. 2011(14)

[3] Yong Yang. Research on Planning Method of Electric Vehicle Fast Charging Station. Master Thesis of North China Electric Power University. 2014

[4] Yunyan Wang, Jiuchun Jiang, Liyong Niu, et al. Management System of Electric Vehicle Charging Station [J]. Microcomputer Development. 2005(11)

[5] Kangyuan Wang, Wuhao Huang, Yu Liu, Yuanping Qian, et al. Application of Design Mode in Software Framework Design of Grid Monitoring System [J]. Automation of Electric Power Systems. 2003(13)

[6]Hui Yan, Gengyin Li, Lei Zhao, Bin Wu, et al. Design and Realization of Monitoring System for Electric Vehicle Charging Station [J]. Power System Technology. 2009(12)

[7]Mingyu Zhao, Gang Wang, Yinghui Wang, Guangming Sun, et al. Design and Realization of Monitoring System for Electric Vehicle Charging Facility [J]. Automation of Electric Power Systems. 2011(10)

[8]Xin Su. Design and Realization of Billing Management System for Electric Vehicle Charging Station. Master Thesis of North China Electric Power University. 2012

[9]Lei Pan, Jiuchun Jiang, Liyong Niu, et al. Communication Network and Interface Design of Electric Vehicle Charging Station [J]. Microcomputer Information. 2005(02)

[10] Jian Wang, Jiuchun Jiang, et al. Design and Realization of Information Management System for Electric Vehicle Charging Station [J]. Microcomputer Information. 2006(15) 